\title{
ROLE OF THE THERMAL STRESS ON THE MAGNETOPHONON PEAK STRUCTURE IN THE PARALLEL TRANSPORT OF THE GaAs/AlGaAs MULTIPLE QUANTUM WELLS
}

\author{
G. Tomaka* , J. Cebulski, E.M. Sheregil, W. Ściuk \\ Institute of Physics of Pedagogical University \\ Rejtana 16a, 35-310 Rzeszów, Poland
}

W. STrupiński and L. DobrzańsKi

Institute of Electronic Materials Technology, Wólczyńska 133, 01-919 Warsaw, Poland

The magnetophonon resonance in parallel transport of two types multiple quantum wells was studied. The transverse magnetoresistance was measured in pulsed magnetic fields up to $30 \mathrm{~T}$ (within temperature region from 77 to $340 \mathrm{~K}$ ). A fine structure of magnetophonon resonance peaks which depends on temperature and does not depend on the type of multiple quantum wells, was observed. This effect could be attributed to two phenomena: contribution of barrier phonons and influence of thermostresses.

PACS numbers: 78.66.Fd, 73.20.Dx

The magnetophonon resonance (MPR) together with the cyclotron resonance has become one of the most widely applied methods of spectroscopy of electron states in semiconducting bulk crystals [1] and singular heterostructures [2]. There are two papers known in which MPR was examined in superlattices and structures with multiple quantum wells (MQW) in the vertical transport (both the magnetic field and the electric current are perpendicular to layers) [3, 4]. The present paper reports results of the MPR research in MQW in the parallel transport (the magnetic field is perpendicular, and the current is parallel to layers). In particular, sensitivity of the phonon spectrum and electron states to thermostresses is considered.

Two types of MQW-systems were studied; they consisted of ten QWs of GaAs and ten AIGaAs barriers, and were obtained by metalorganic chemical vapor deposition (MOCVD) on semi-insulating GaAs substrate. The parameters of MQWs are shown in Table. Six specimens in standard form for Hall measurements

*e-mail: gmtomaka@univ.rzeszow.pl 


\section{TABLE}

Parameters of samples.

\begin{tabular}{c|c|c|c}
\hline \hline Samples & $L_{\text {GaAs }}[\AA] / L_{\text {AlGaAs }}[\AA]$ & $x$ & Carrier density $\left[10^{10} \mathrm{~cm}^{-2}\right]$ \\
\hline 151 & $100 / 40$ & 0.3 & 50 \\
152 & $100 / 40$ & 0.3 & 5 \\
153 & $80 / 40$ & 0.3 & 50
\end{tabular}

obtained by photolithography from the sample $151 \mathrm{MQWs}$ (see Table) were measured as well as three and two specimens from 152 and 153 samples, respectively.

The MPR research was performed in pulsed magnetic fields up to $30 \mathrm{~T}$. A transient recorder was used to store transverse magnetoresistance $\rho_{x x}(B)$ and a voltage $\approx \partial B / \partial t$ which was numerically integrated to find the $\boldsymbol{B}$ - magnetic field induction. The transverse magnetoresistance was measured between $77 \mathrm{~K}$ and $340 \mathrm{~K}$ and the MPR oscillations extracted by subtracting a voltage linear in magnetic field. The oscillating part of magnetoresistance $\Delta \rho_{x x}$ was recorded.

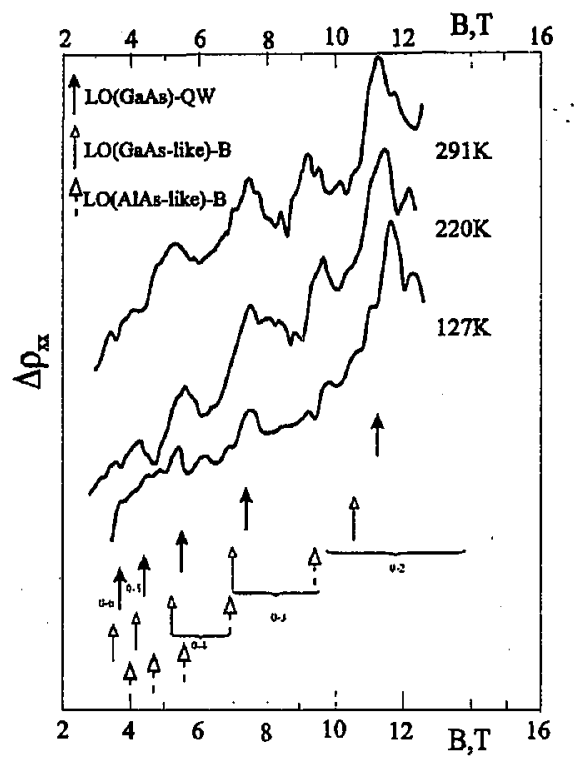

Fig. 1. Experimental recordings of $\Delta \rho_{x x}(B)$ (arb. units) obtained for sample 153 at three different temperatures.

In Figs. 1-3 the examples of registered curves $\Delta \rho_{x x}(B)$ are shown where the total structure of the observed peaks was represented due to remarkable repetition for different MPR harmonics at different temperatures.

Since the repetition of MPR peaks structure for different harmonics and given MQW is especially pronounced in the range of magnetic fields $0-16 \mathrm{~T}$, the resonance curves just for that range of magnetic field are represented in Figs. 1-3. 
Role of the Thermal Stress on the Magnetophonon Peak Structure ... 599

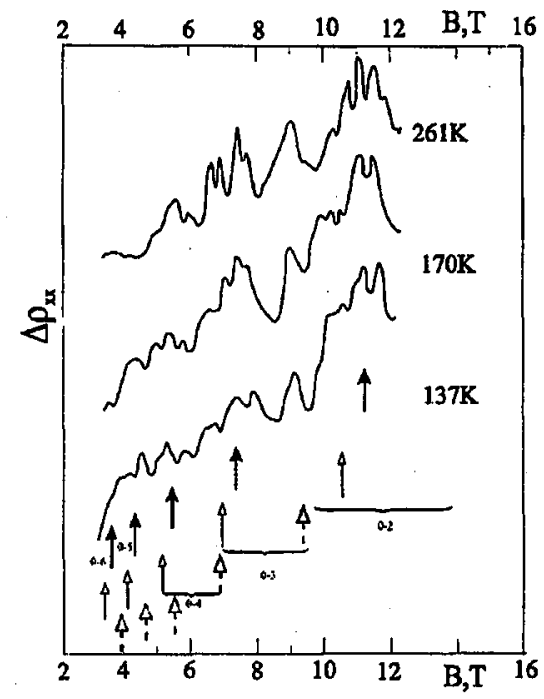

Fig. 2. Experimental recordings of $\Delta \rho_{x x}(B)$ (arb. units) obtained for sample 151 at three different temperatures.

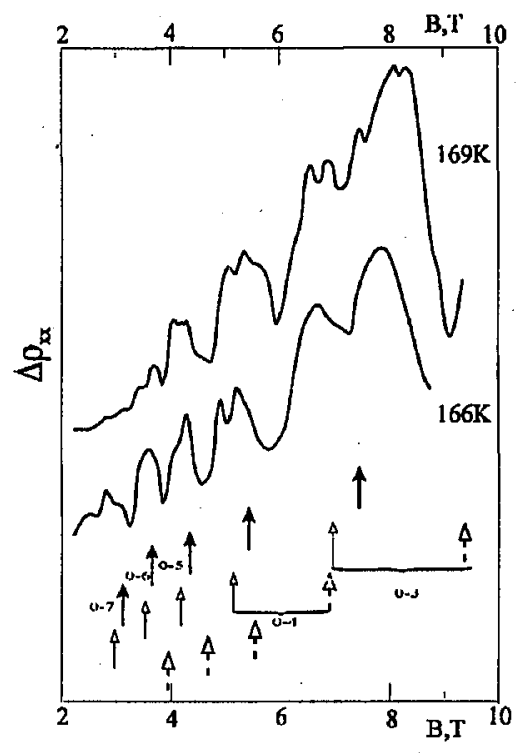

Fig. 3. Experimental recordings of $\Delta \rho_{x x}(B)$ (arb. units) obtained for sample $152(T=$ $166 \mathrm{~K})$ and sample $151(T=169 \mathrm{~K})$.

Representing the resonance curves in larger scale of magnetic fields up to $30 \mathrm{~T}$ (including 0-1 transition), make impossible to observe this repetition of peak structure for different harmonics. 
MPR spectra obtained at three temperatures for the sample 153 are shown in Fig. 1 and for the sample 152 - in Fig. 2. Figure 3 represents two curves obtained at two closed temperatures for the samples 151 and 152 . In these figures the next regularities can be seen:

1. the structure of peaks tends to repeat its structure in consecutive harmonics for the given sample at different temperatures;

2. the observed structure is characteristic of both the examined specimen and of the given MQW type;

3. the fine structure of MPR peaks reappears in each type of MQW, but its character is not the same.

It follows that the occurrence of the observed peak structure depends neither on the two-dimensional density of carriers, nor on the size of the QW. Raising the temperature of the measurement the fine peak structure becomes more clearly defined. This can be seen in Fig. 2 as the temperature evolution of peaks $0-2$ and $0-3$, as well as in Fig. 1, for the transition 0-3.

To facilitate the interpretation of the observed peak structure, in Figs. 1-3 by thicker arrows the positions of MPR peaks observed in the single heterostructure $\mathrm{GaAs} / \mathrm{Al}_{x} \mathrm{Ga}_{1-x}$ As at the temperature $130 \mathrm{~K}$, are indicated [5]. In Ref. [5], for the case of MPR in the single heterostructure each transition between Landau levels corresponded to one structureless peak and at the some time the resonance fields were somewhat smaller than theoretical values determined by the condition

$$
N \hbar \omega_{\mathrm{C}}=\hbar \omega_{\mathrm{LO}}(\mathrm{GaAs}), \quad N=1,2,3 \ldots
$$

where the longitudinal optical (LO)-phonon is the bulk GaAs LO-phonon.

During the observation of MPR in vertical transport in a superlattice where electrons move in minibands, the authors [3] have not noticed any extra peak structure but only disappearance of some of them caused by the imposition (on condition (1)) of an additional restriction linked with the minibands' width. Whereas in [4], where examination of MPR in the vertical transport in MQW GaAs/AIAs was reported, besides MPR oscillations connected with the absorption of the QW GaAs LO-phonons also peaks caused by interaction of electrons with LO-phonons of the AlAs barrier occur. In Figs. 1-3 thin and dashed arrows indicate what resonance field would correspond to resonances caused by absorption of phonons of the $\mathrm{Al}_{0.3} \mathrm{Gaa}_{0.7} \mathrm{As}$ barrier ${ }^{\dagger}$ (LO GaAs-like and LO AlAs-like respectively) with transitions between Landau levels just like in the main series, caused by the interaction with phonons of the GaAs QW. The partly observed peak structure can be explained by the introduction to MPR two additional types of barrier phonons. Therefore, the occurrence of peak at about $9 \mathrm{~T}$ can be explained by the transition 0-3 of an electron with the absorption AlAs-like LO phonon. The MPR caused by interaction between an electron and phonons from the barrier made by GaAs-like lattice manifest themselves as satellites of each main series peaks, to the side of smaller magnetic fields. The last one is mostly visible in Fig. 3, where the fine structure is less prominent.

\footnotetext{
t The frequencies of $\mathrm{LO}$-phonons of $\mathrm{Al}_{0.3} \mathrm{Ga}_{0.7} \mathrm{As}$ are taken from Ref. [6].
} 
Stresses in GaAs layers caused by mismatch between the QW lattice and the barrier should be accounted for when the measurement temperature is lower than the temperature of creation of the layers with MOCVD technology. Mismatch leads to a tension in the plane of GaAs layers. Because of their various distributions in the MQW their contribution, sign included, into the shift of the resonance field will vary. It is the effect that can explain the disintegration of magnetoconductivity in MQW during parallel transport. Let us estimate then the magnitude of shift of the resonance fields caused by thermostresses in GaAs layers. At the temperature of $77 \mathrm{~K}$ the mismatch between $\mathrm{GaAs}$ and $\mathrm{Al}_{0.3} \mathrm{Ga}_{0.7}$ As lattices has the value $\Delta a=4 \times 10^{-13} \mathrm{~m}[6]$, which makes the biaxial deformation $\varepsilon_{0}$ in the plane of the layer equal to $7.1 \times 10^{-4}$. Based on considerations in [7] and [8] we can conclude that the overall change of the energy gap caused by those stresses can be determined as

$$
\Delta E_{\mathrm{g}}=\Delta E_{\mathrm{hy}}^{\mathrm{v}}+\Delta E_{\mathrm{an}}^{\mathrm{v}}-\Delta E_{\mathrm{hy}}^{\mathrm{c}},
$$

where $\Delta E_{\text {hy }}^{\mathrm{v}}$ is the displacement of valence band caused by the hydrostatic stress component [8], $\Delta E_{\mathrm{hy}}^{\mathrm{c}}$ is the same for the conduction band, sign included and is twice larger than $\Delta E_{\mathrm{hy}}^{\mathrm{v}}[7], \Delta E_{\mathrm{an}}^{\mathrm{v}}$ is the contribution to the displacement of the light holes valence band caused by the biaxial stress component [8]. The next values of the potential of overall deformation $a$, the potential of uniaxial deformation $b$

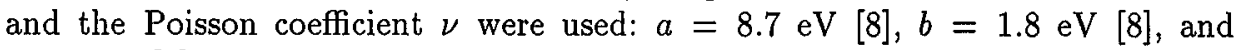
$\nu=0.31[6]$. Therefore, these components have the following values: $\Delta E_{\mathrm{hy}}^{\mathrm{v}}(\varepsilon)=$ $6.707 \mathrm{meV}, \Delta E_{\mathrm{am}}^{\mathrm{v}}(\varepsilon)=2.392 \mathrm{meV}, \Delta E_{\mathrm{hy}}^{\mathrm{c}}(\varepsilon)=-13.415 \mathrm{meV}$, and the overall change of the energy gap is $\Delta E_{\mathrm{g}}=22.5 \mathrm{meV}$. The change of energy gap by $1.5 \%$ caused by thermostresses is accompanied by a change of effective mass at the bottom of the conduction band, which is about $1.4 \%$ if one takes into account a simple three-band model. The change of effective mass causes the shift of MPR peaks to the smaller magnetic fields of about $1.4 \%$.

In addition, anisotropic stresses cause phonon frequencies to shift and even the LO phonon branches to split [9]. Therefore the maximum decrease in LO phonons frequency in the GaAs layer induced by biaxial stress can be evaluated by the formula [10]: $\Delta \omega_{\mathrm{LO}}=-4.8 \times 10^{2} \varepsilon_{0}\left[\mathrm{~cm}^{-1}\right]$. In our case, a decrease in the LO phonon frequency is equal to $0.33 \mathrm{~cm}^{-1}$, that is $0.1 \%$.

For those reasons, changes of $E_{\mathrm{g}}$ and $\mathrm{LO}$ frequencies should be shifted in the same direction (i.e. to the lower magnetic fields). This shift should not be greater than $1.5 \%$. It means that for example in the case of peak at $11.2 \mathrm{~T}(0-2$ transition $)$ the maximum split caused by disintegration of magnetoconductivity in the parallel transport resulting from thermostresses distribution in the layers, is of $0.17 \mathrm{~T}$. If the satellite of this peak (caused by the interaction with the LO phonon of the GaAs-like lattice barrier) is taken into account, then in this magnetic field range (10-11 T) three additional peaks are expected. Such a structure should reappear for other MPR harmonics. This can partly (for some curves) explain the observed peak structure. 


\section{References}

[1] Y.A. Firsov, V.L. Gurevich, R.V. Parfeniev, I.M. Tsidilkovskii, in: Landau Level Spectroscopy, Eds. G. Landwehr, E.I. Rashba, in series Modern Problems in Condensed Matter Sciences, Eds. V.M. Agranovich, A.A. Maradudin, Vol. 27.2, North-Holland, Amsterdam 1991, p. 1181.

[2] R.J. Nicholas, in Ref. [1], p. 777.

[3] H. Nogutchi, H. Sakaki, T. Takamasu, N. Miura, Phys. Rev. B 45, 12148 (1992).

[4] P. Gassot, J. Genoe, D.K. Maude, J.C. Portal, K.S.H. Dalton, D.M. Symons, R.J. Nicholas, F. Arstone, J.F. Palmier, F. Lauelle, Phys. Rev. B 54, 14540 (1996).

[5] M.A. Brummel, R.J. Nicholas, M.A. Hopkins, Phys. Rev. Lett. 58, 77 (1987).

[6] Aluminium Gallium Arsenide, Ed. S. Adachi, in series Emis Datareviews No. 7, INSPEC, Institution of Electrical Engineers, London 1993.

[7] S.L. Chuang, Phys. Rev. B 43, 9649 (1991).

[8] T.P. Sosin, W. Trzeciakowski, Acta Phys. Pol. A 87, 151 (1995).

[9] P. Wickboldt, E. Anastassakis, R. Sauer, M. Cardona, Phys. Rev. B 35, 1362 (1987).

[10] P.P. Lottici, G. Attolini, E. Chimenti, C. Pelosi, Solid State Commun. 99, 537 (1996). 